\title{
Coupled dynamics of heavy and light flavor flow harmonics from EPOSHQ
}

\author{
P.B. Gossiaux ${ }^{1, \star}$, J. Aichelin ${ }^{1}$, M. Nahrgang ${ }^{1}$, V. Ozvenchuk ${ }^{1,2}$, and K. Werner ${ }^{1}$ \\ ${ }^{1}$ SUBATECH, UMR 6457, IMT Atlantique, Université de Nantes, IN2P3/CNRS. \\ 4 rue Alfred Kastler, 44307 Nantes cedex 3, France \\ ${ }^{2}$ Cracow, INP, ul. Radzikowskiego 152, 31-342 Kraków, Poland
}

\begin{abstract}
We pursue the study of event by event correlations between low-mass particles and heavy mesons flow harmonics in ultrarelativistic heavy ion collisions and clarify some ambiguities found in one of our previous work.
\end{abstract}

\section{Introduction and method}

The joint study of light and heavy particles produced in ultrarelativistic heavy ion collisions offers new insights into several physical aspects, in particular into the coupling of heavy quarks with the quark gluon plasma (QGP) formed in these collisions. In [1], we have focused on the comparison between flow harmonics of light and heavy mesons and conjectured that heavy mesons would benefit less from the bulk flow due to their inertia. Such an effect could f.i. manifest itself in event by event (EBE) correlations between those flow harmonics, as studied recently in [2] and [3], where anomalous fluctuations where found.

In this study, we follow the same method as in [3], using the EPOSHQ model which allows to describe and predict both, the light and the heavy, sectors in ultrarelativistic pp, pA and AA collisions. Heavy quarks strongly interact with the QGP and thus benefit from the collective flows of the bulk. Later, D mesons are allowed to interact with light hadrons in the hadronic phase, leading to some moderate extra flow. For the sake of simplicity, D mesons are assumed to be produced exclusively through fragmentation mechanism while the role of coalescence will be investigated in an upcoming study. The analysis is performed EBE using the event/participant plane method, which does not suffer from the usual caveats as the participant plane is known with a very good accuracy from the initial state conditions. In [3], we have artificially enhanced the c quark production by a factor 50 in order to reduce the $v_{n}$ fluctuations resulting from the limited number of c quarks [4]. This, however, seems to lead to abnormally large fluctuations and even led us to conjecture the existence of large EBE fluctuations specific to heavy flavor production and propagation in QGP. In this work, we do not resort to such an oversampling.

\footnotetext{
^e-mail: gossiaux@ subatech.in2p3.fr
} 


\section{Results and analysis}

In the following, "^" symbols will be used for EBE quantities. In fig. 1, we show the distribution of the $\hat{v}_{n=2,3}$ obtained $^{1}$ for $\pi, \mathrm{p}, \mathrm{c}$-quarks (before hadronization) and D mesons in EPOSHQ events, taking $\hat{\Psi}_{n}^{\mathrm{PP}}$ as the EBE reference plane for all species. The large variances observed for the flows of HF mesons imply that their individual EBE flows are often found with opposite sign as compared to the average. Following [4], we expect the variance of $\hat{v}_{n=2,3}$ to be $\operatorname{var}\left(\hat{v}_{n}\right) \approx \frac{1}{2 N}+\operatorname{var}_{\mathrm{EBE}, n}$. In table 1, we provide the numbers associated to the various cases shown in fig. 1 . We observe a clear transition from $\pi$ (dominance of EBE fluctuations) $\rightarrow \mathrm{N} \rightarrow \mathrm{D}$, for which the fluctuations are quite well explained by the usual $1 / 2 \mathrm{~N}$ term, in contrast with our previous observation [3]. We have checked that this also holds for other centrality classes, other energies, larger HF coupling to the QGP bulk, finite $p_{T}$ cut off, etc.
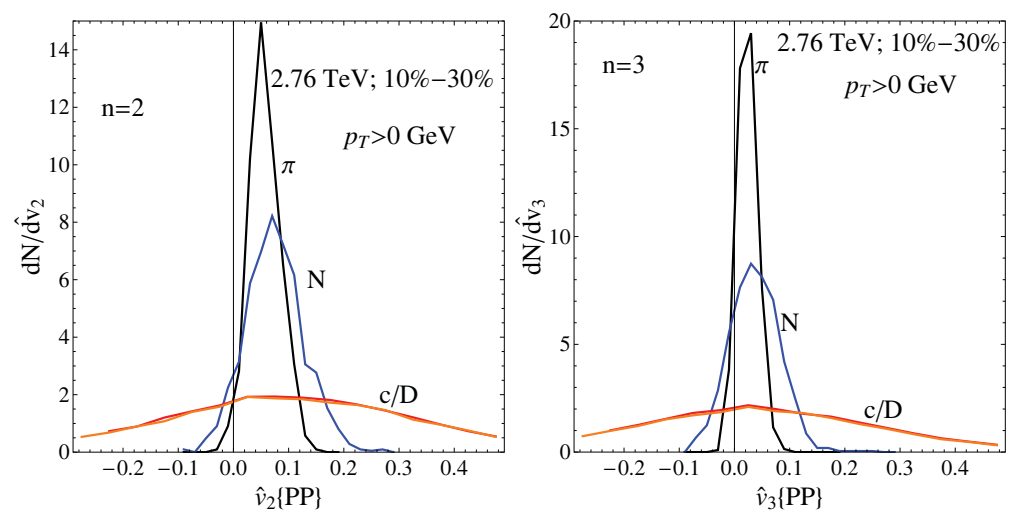

Figure 1. The distributions of the elliptic (left) and triangular (right) flow of final pions, nucleons (N), charmed quarks and $\mathrm{D}$ mesons produced in $\mathrm{Pb}-\mathrm{Pb}$ collisions at $\sqrt{s}=2.76 \mathrm{TeV}$ in the $10 \%-30 \%$ centrality class. Distributions of $\mathrm{c}$ quarks and $\mathrm{D}$ mesons are quite similar.

Table 1. Decomposition of the flow variance for the 10\%-30\% centrality class.

\begin{tabular}{ccccc}
\hline & $\operatorname{var}\left(\hat{v}_{2 / 3}\right)$ & $\left\langle\frac{1}{2 \hat{N}}\right\rangle$ & residual $\left(\operatorname{var}\left(\hat{v}_{2 / 3}\right)-\left\langle\frac{1}{2 \hat{N}}\right\rangle\right)$ & $\%$ age of fluct. due to $\left\langle\frac{1}{2 \hat{N}}\right\rangle$ \\
\hline$\pi$ & $8.2 \mathrm{E}-4 / 3.3 \mathrm{E}-4$ & $1.3 \mathrm{E}-4$ & $6.9 \mathrm{E}-4 / 2 \mathrm{E}-4$ & $16 \% / 39 \%$ \\
$\mathrm{~N}$ & $2.9 \mathrm{E}-3 / 2.2 \mathrm{E}-3$ & $1.8 \mathrm{E}-3$ & $1.1 \mathrm{E}-3 / 4 \mathrm{E}-4$ & $62 \% / 82 \%$ \\
$\mathrm{D}$ & $8.5 \mathrm{E}-2 / 8 \mathrm{E}-2$ & $7.9 \mathrm{E}-2$ & $5.7 \mathrm{E}-3 / 1.6 \mathrm{E}-3$ & $93 \% / 99 \%$ \\
\hline
\end{tabular}

In fig. 2, we show the elliptic and triangular flow of $\mathrm{D}$ mesons as a function of the spatial eccentricities $\hat{\epsilon}_{n}$, in a similar way as our QM2014 approach [5]. A linear increase of $\left\langle\hat{v}_{n}\right\rangle$ with $\left\langle\hat{\epsilon}_{n}\right\rangle$ in the bulk part of the event distribution implies $\operatorname{var}_{\mathrm{EBE}, n} \approx\left(\left\langle\hat{v}_{n}\right\rangle /\left\langle\hat{\epsilon}_{n}\right\rangle\right)^{2} \operatorname{var}\left(\hat{\epsilon}_{n}\right)$, i.e. $\operatorname{var}_{\mathrm{EBE}, 2} \approx 6 . \mathrm{E}-4 / 1.2 \mathrm{E}-3 / 6 \mathrm{E}-4$ for $\pi / \mathrm{N} / \mathrm{D}$ mesons. These numbers should be compared with the 3 rd column of table 1 , with excellent agreement for $\pi$ and nucleons. For HF, an extra source of fluctuations cannot be excluded, but it is definitively much smaller that what was advocated in [3] and is superseded by the $1 / 2 N_{D}$ contribution for practical purposes.

\footnotetext{
${ }^{1}$ According to $\hat{v}_{n}\{\mathrm{PP}\}=\frac{1}{\mathrm{~N}} \sum_{\mathrm{i}=1}^{\mathrm{N}} \cos \left(\mathrm{n}\left(\phi_{\mathrm{i}}-\hat{\mathrm{Y}}_{\mathrm{n}}^{\mathrm{PP}}\right)\right)$, where $N$ is the number of selected particles in the sample and $\phi_{i}$ the azimuthal angles of individual particles.
} 

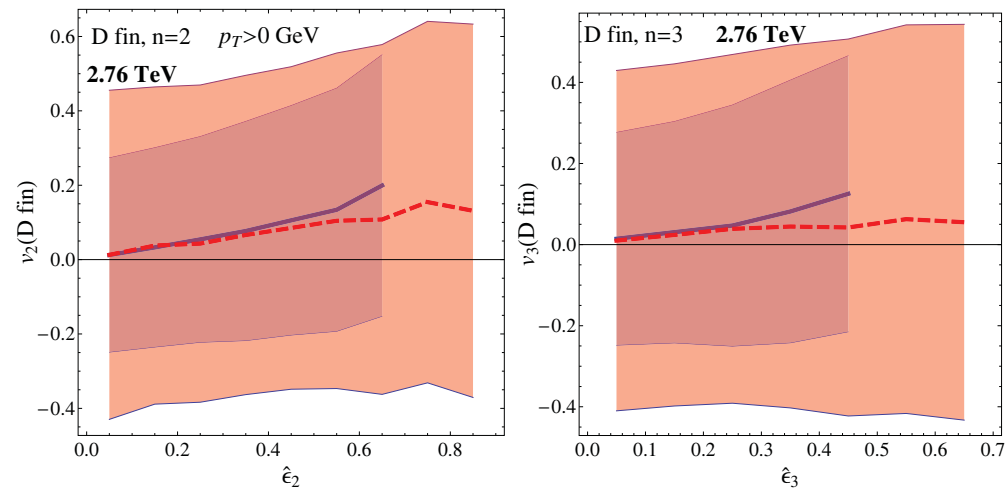

Figure 2. The elliptic (left) and triangular (right) flow of final $\mathrm{D}$ mesons produced in $\mathrm{Pb}-\mathrm{Pb}$ collisions at $\sqrt{s}=$ 2.76 TeV as a function of spatial eccentricity; the solid lines correspond to average values (plain for $10 \%-30 \%$ centrality class and dashed for $30 \%-50 \%$ centrality class). The red (30\%-50\%) and darker (10\%-30\%) bands represent the average $\pm 1 \sigma$ interval.
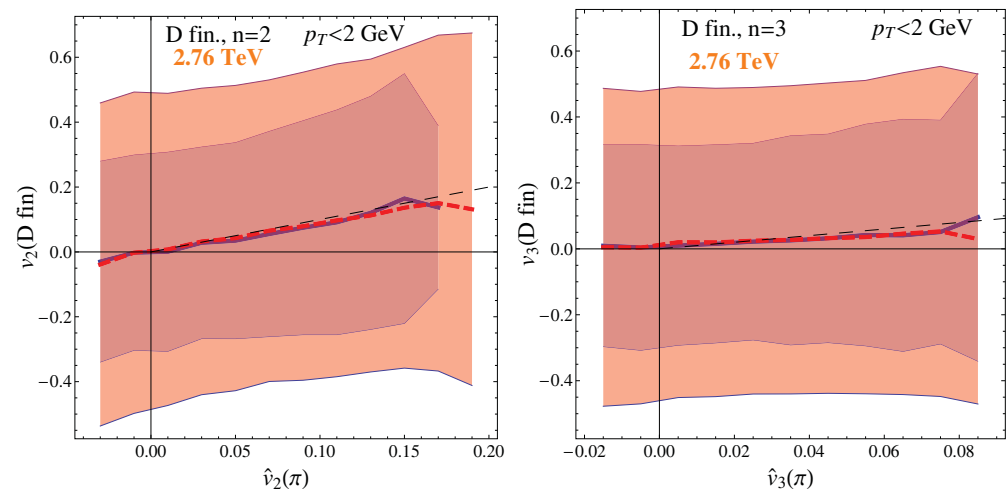

Figure 3. The elliptic (left) and triangular (right) flow of final $p_{T}<2 \mathrm{GeV} \mathrm{D}$ mesons produced in $\mathrm{Pb}-\mathrm{Pb}$ collisions at $\sqrt{s}=2.76 \mathrm{TeV}$ as a function of equivalent quantity for $\pi$; same conventions as for fig. 2 . The thin dashed line represents the $\left\langle\hat{v}_{n}(D)\right\rangle=\left\langle\hat{v}_{n}(\pi)\right\rangle$ case.

We have performed a refined analysis to identify the origin of the large fluctuations found previously with the oversampling method. In practice such an oversampling is achieved by performing 50 so-called "HQ events" - i.e. evolution of the HQ in the bulk - for a single EPOS3 global event with the same initial heavy quarks. This is an unavoidable feature as the production of heavy-quarks is deeply rooted in the semi-hard pomeron approach of EPOS3. The 50 replications of the HQ $\hat{v}_{n}$ are therefore totally correlated at initial time and gradually decorrelate with the time evolution. During our previous analysis, we had taken care to check that a very good level of decorrelation is achieved at the end of the evolution. However, we have realized since then that a small persisting correlation generates, in turn, an extra contribution in $\operatorname{var}\left(\hat{v}_{n}\right)$ that scales like the number of replications and thus appears as a contribution to $\operatorname{var}_{\mathrm{EBE}, n}$. This explains our misinterpretation in [3].

Having the fluctuations under control, we focus on the averages $\left\langle\hat{v}_{n}\right\rangle$ in fig. 2. Our results are compatible with the conclusions of our study [1] performed with EPOS2 as a description of the bulk: 
The higher the eccentricities, the harmonics (and the mass) the less heavy quarks benefit from the bulk flow. In fig. 3 and 4, we present the correlation between the flow of low $p_{T}$ final pions ${ }^{2}$ and the equivalent flow for low and intermediate $p_{T}$ D-mesons. In the low $p_{T}$ case - where one expects nearly perfect thermalization of heavy quarks -, one finds a very good correlation between the light and the heavy sector. For intermediate $p_{T}$ D-mesons, the correlation is still quite stong but less pronounced, which is an indication that $c$ quarks with finite $p_{T}$ are slightly less coupled with the bulk. Equivalent studies with $b$ quarks are underway.
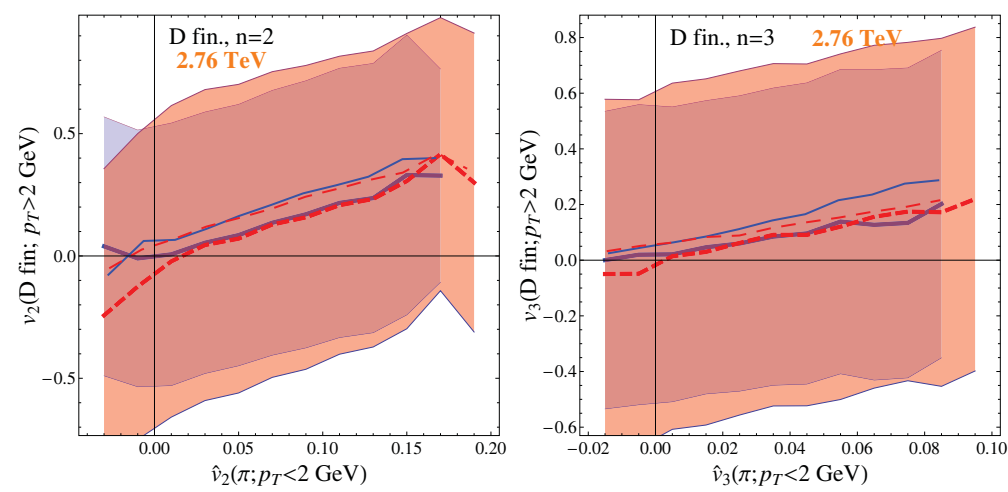

Figure 4. Same as fig. 3 for D mesons with $p_{T}>2 \mathrm{GeV}$; thin lines show the corresponding flows for pions with $p_{T}>2 \mathrm{GeV}$ for comparison.

\section{Conclusions}

We have refined our analysis of heavy-light flow correlations initiated in [3] in two major aspects: First, we have understood that the HQ oversampling procedure leads to spurious extra-fluctuations in the EPOSHQ framework and thus clarified the ambiguities raised in [3]. Although some specific EBE fluctuations of the HF harmonic-flow cannot be ruled out, they appear to be subdominant. Second, we have increased our samples and present more robust predictions for the correlations between D mesons and pions harmonic flows. These could be measured experimentally by event engineering techniques, triggering on the $\hat{v}_{n}$ of pions either in minimum bias events or for a given centrality class. Going towards larger $\sqrt{s}$ facilitates this study, as the fluctuations are reduced due to the more abundant HQ production.

\section{References}

[1] M. Nahrgang et al., Phys. Rev. C91, 014904 (2015)

[2] C.A.G. Prado et al, arxiv:1611.02965

[3] P.B. Gossiaux et al., Nucl. Phys. A967, 672 (2017)

[4] A. Poskanzer and S. Voloshin, Phys. Rev. C58, 1671 (1998)

[5] https://indico.cern.ch/event/219436/contributions/1523417/

\footnotetext{
${ }^{2}$ Taken as the best proxy to probe the initial eccentricities of the $\mathrm{Pb}-\mathrm{Pb}$ collisions.
} 